\title{
Radiative hydrodynamic simulations of acoustic waves in sunspots
}

\author{
S. Bard \& M. Carlsson \\ Institute of Theoretical Astrophysics, University of Oslo, P.O. box 1029 Blindern, N-0315 Oslo, \\ NORWAY
}

\begin{abstract}
We describe fully consistent NLTE radiation hydrodynamic simulations of the propagation of acoustic waves in sunspot umbrae. In contrast to the case of the quiet internetwork Sun, we find that the observed behavior of the resonance lines of singly ionized calcium $(\mathrm{H}$ and $\mathrm{K}$ lines) cannot be explained without adding non-acoustic heating. The best agreement with observations is obtained with an extra heating term as a function of height that is constant per volume.
\end{abstract}

This study is motivated by shock signatures observed above sunspot umbrae as shown in figure 1 from Bard \& Carlsson (1997). The Ca II K line intensity is shown (figure 1,
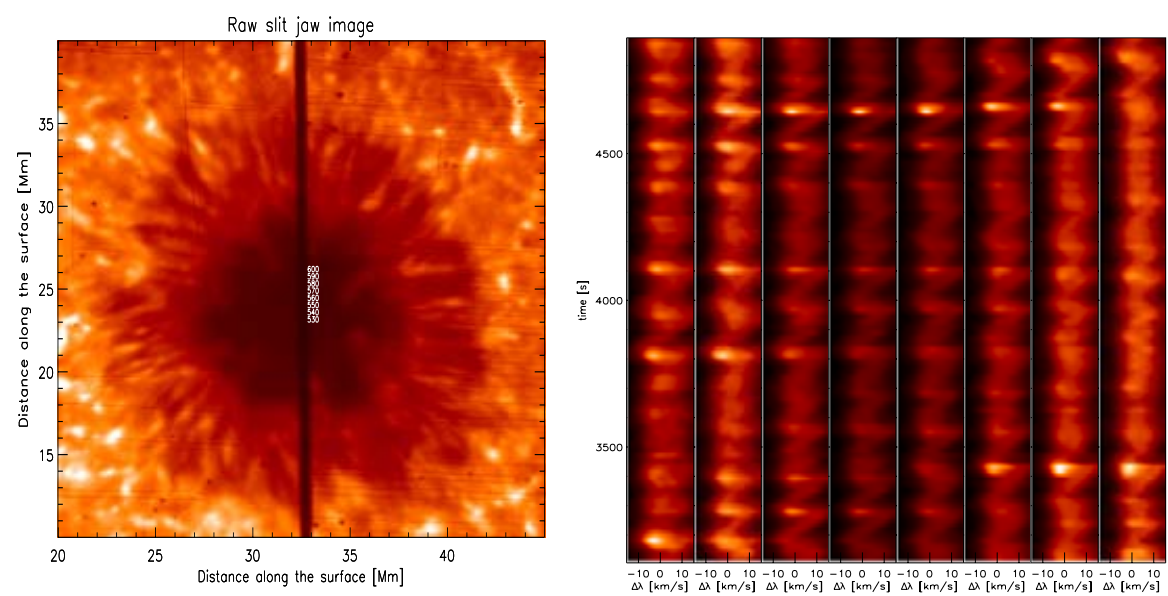

Figure 1: Left: slit jaw image. Right: observed intensity evolution.

right) as a function of wavelength and time. Each time sequence corresponds to a single point along the slit (figure 1, left). The rapid shift of the line core toward the blue followed by the gradual return to the red which produces the characteristic sawtooth shape is usually attributed to shock propagation through the region. We examine different heating mechanisms using a grid of initial temperature structures (figure 2, top). SPOTM refers to the temperature structure from model M from Malby et al. (1986). The computed Ca II K line intensity as a function of wavelength and time for five simulations are shown in figure 2 (bottom). The left most panel shows simulation with no extra heat input. The right most panel, with constant heat per $\mathrm{cm}^{3}$ input. SPOTMHeI, SPOTMHeII and SPOTMHeII have increasing amounts of constant heat per mass input. The important feature of emission between shocks, present in observations (figure 1), is only present in the simulations were extra heat is input. Also the best agreement with observations is obtained in simulations where extra heat input is constant per volume (SPOTMHeV). 


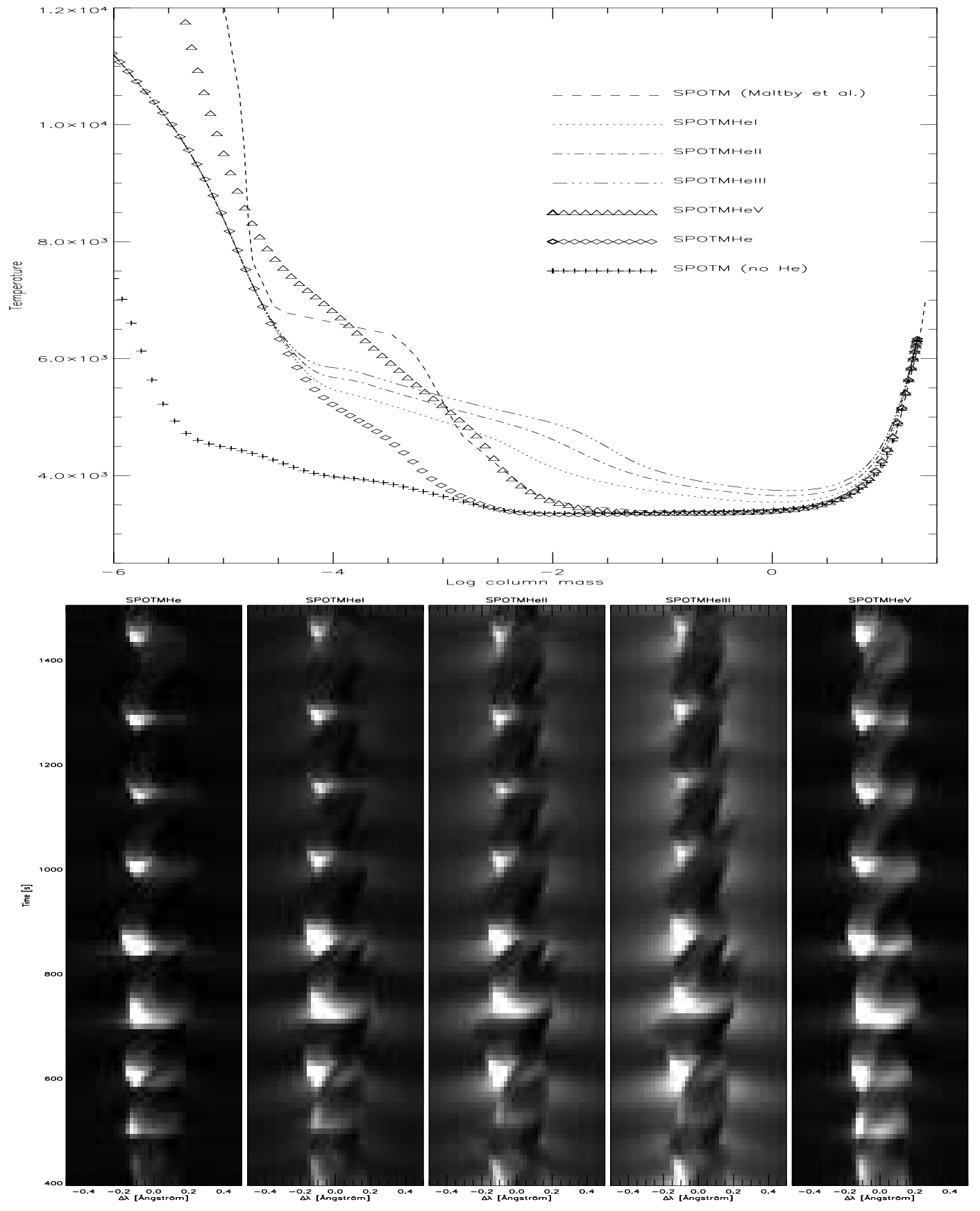

Figure 2. Top: initial temperature structures. Bottom: simulated intensity evolution.

\section{References}

Bard S. \& Carlsson M. 1997 ESA SP-404: Fifth SOHO Workshop SP-404 189-193

Maltby P., Avrett E. H., Carlsson M., Kjeldseth-Moe O., Kurucz R. L., \& Loeser R. 1986 Astrophysical Journal 306, 284-303. 